\title{
Holmium Laser Enucleation of the Prostate is Safe for Patients Above 80 Years: A Prospective Study
}

\author{
Songzhe Piao ${ }^{1,2}$, Min Soo Choo ${ }^{3}$, Myong $\mathrm{Kim}^{1}$, Ho Joon Jeon ${ }^{1}$, Seung-June $\mathrm{Oh}^{1}$ \\ ${ }^{1}$ Department of Urology, Seoul National University Hospital, Seoul, Korea \\ ${ }^{2}$ Department of Urology, Yanbian University Hospital, Yanji, China \\ ${ }^{3}$ Department of Urology, Dongtan Sacred Heart Hospital, Hwaseong, Korea
}

Purpose: To evaluate the effect of age on the efficacy and safety of holmium laser enucleation of the prostate (HoLEP) for the treatment of symptomatic benign prostatic hyperplasia $(\mathrm{BPH})$.

Methods: A total of 579 patients underwent HoLEP procedure performed by a single surgeon (SJO) between December 2009 and May 2013. The perioperative and functional outcomes of patients in the age groups of 50-59 (group A, $n=44$ ), 60-69 (group B, $\mathrm{n}=253$ ), 70-79 (group C, $\mathrm{n}=244$ ), and $\geq 80$ years (group D, $\mathrm{n}=38$ ) were compared. The Clavien-Dindo system was used to evaluate clinical outcomes. The International Prostate Symptom Score (IPSS), maximum urinary flow rate (Qmax), postvoid residual (PVR) urine volume, and urinary continence were used to assess functional outcomes.

Results: In this study, the patients $\geq 80$ years had significantly higher presence of hypertension $(\mathrm{P}=0.007)$, total prostate volumes $(\mathrm{P}=0.024)$, transitional zone volume $(\mathrm{P}=0.002)$, American Society of Anesthesiologists scores $(\mathrm{P}=0.006)$, urinary retention $(\mathrm{P}=0.032)$, and anticoagulation use $(\mathrm{P}=0.008)$ at preoperative period. Moreover, the mean values of operation time, enucleation time, morcellation time, and enucleation weight were higher in group $\mathrm{D}$ compared with other group patients $(\mathrm{P}=0.002, \mathrm{P}=0.010, \mathrm{P}<0.01$, and $\mathrm{P}=0.009$, respectively). Patients aged $\geq 80$ years had a longer hospital stay time $(2.9 \pm 1.8$ days) than other groups (group $A, 2.3 \pm 0.7$ days; group $B, 2.3 \pm 0.7$ days vs. group $C, 2.4 \pm 0.7$ days; $P=0.001$ ). All groups were similar in regard to the incidence of complications (Clavien-Dindo grade) post operatively $(\mathrm{P}>0.05)$. All the patients in the present study showed improvement in functional outcomes after HoLEP. By the sixth month, there were no significant differences in IPSS, quality of life, Qmax, and PVR among the groups $(\mathrm{P}>0.05)$.

Conclusions: Compared with younger patients, the patients aged $\geq 80$ years had a similar overall morbidity and 6-month functional outcomes of HoLEP. HoLEP is a safe and effective treatment for BPH among the elderly.

Keywords: Laser Therapy; Prostate; Prostatic Hyperplasia; Transurethral Resection of Prostate

- Research Ethics: All data were collected prospectively using a planned data registry form from the Seoul National University Hospital (SNUH) BPH Prospective Database Registry. The Institutional Review Board of SNUH approved the study design and the use of patient information extracted from the hospital database (approval number: H-1509-094-705). Written informed consents from the patients were not required. This trial was performed in accordance with the Declaration of Helsinki.

- Conflict of Interest: No potential conflict of interest relevant to this article was reported.

Corresponding author: Seung-June Oh (iD http://orcid.org/0000-0002-0322-3539 Department of Urology, Seoul National University Hospital, 101 Daehak-ro, Jongno-gu, Seoul 03080, Korea

E-mail: sjo@snu.ac.kr / Tel: +82-2-2072-2406 / Fax: +82-2-742-4665

Submitted: November 22, 2015 / Accepted after revision: January 27, 2016
This is an Open Access article distributed under the terms of the Creative Commons Attribution Non-Commercial License (http://creativenses/by-nc/4.0/) which permits unrestricted non-commercial use, distribution, and reproduction in any medium, provided the original work is properly cited. 


\section{INTRODUCTION}

Benign prostatic hyperplasia (BPH) is a common cause of lower urinary tract symptoms (LUTS) and bladder outlet obstruction in elderly men and has a significant impact on their healthrelated quality of life (QoL) [1]. The increasing aging population has a large number of elderly patients with symptomatic BPH considered for surgery after the failure of conservative medical therapies [2].

Several minimally invasive procedures for treating $\mathrm{BPH}$ have been developed. Transurethral resection of the prostate (TURP) is considered as the "gold standard" for the treatment of symptomatic $\mathrm{BPH}$. However, some elderly patients with $\mathrm{BPH}$, who often have one or more significant comorbidities, such as severe hypertension, myocardial infarction, liver and kidney dysfunction, diabetes, or the presence of a cardiac pacemaker may not be suitable candidates for TURP. This is particularly true as the risk of comorbidities grows exponentially with age [3]. In recent decades, holmium laser enucleation of the prostate (HoLEP) is being increasingly used as a safe, reproducible, and effective surgical modality for the management of $\mathrm{BPH}$ independent of the prostate size [3]. In recent times, there is an increased amount of evidence that supports the safety and efficacy of HoLEP for BPH when compared to other surgical treatments such as an open prostatectomy (OP) and TURP [3]. Therefore, HoLEP is poised to replace all of these modalities as the new "gold standard" for surgical management of BPH [4]. However, the effect of age on the efficacy and morbidity of HoLEP is poorly known. The aim of this study was to evaluate the effect of age on the efficacy and safety of HoELP.

\section{MATERIALS AND METHODS}

\section{Patients and Study Design}

Between December 2009 and May 2013, 579 patients who underwent HoLEP for symptomatic BPH by one surgeon (SJO) were recruited into the study at the Department of Urology, Seoul National University Hospital (SNUH). All consecutive patients were included in the analysis without any exclusion to avoid potential selection bias. All data were collected prospectively using a planned data registry form from the SNUH BPH Prospective Database Registry. The Institutional Review Board (IRB) number of the SNUH is H-0810-027-260.

The inclusion criteria at the initial time of registration were: (1) patients aged over 50 years with $\mathrm{BPH}$; (2) patients who were diagnosed with BPH by clinical symptoms and correlated examination. All patients were evaluated before surgery by history taking, physical examination, American Society of Anesthesiologists (ASA) score, International Prostate Symptom Score (IPSS), QoL, uroflowmetry (UFM), post-void residual (PVR) urine volume measurement, serum prostate-specific antigen (PSA), and transrectal ultrasonography (TRUS). A multichannel urodynamic study (MMS UD-2000, Medical Measurement System, Enschede, The Netherlands) was performed to help differentiate a bladder outlet obstruction and detrusor overactivity. The IRB of SNUH approved the study design and the use of patient information extracted from the hospital database (approval number: H-1509-094-705). Written informed consents from the patients were not required. This trial was performed in accordance with the Declaration of Helsinki.

\section{Surgical Methods}

The HoLEP procedures used were as previously described [3]. In brief, a "three-lobe" technique was used. A 26-Fr resectoscope (Karl Storz GmbH \& Co, Tuttlingen, Germany) was inserted into the prostate and bladder. Enucleation of the prostate was performed using a $550-\mu \mathrm{m}$ end-firing laser fiber (SlimLine, Lumenis Ltd, Yokneam, Israel) and an 80-W holmium neodymium: yttrium-aluminum-garnet laser (VersaPulse PowerSuite, Lumenis Ltd). The laser setting was $2 \mathrm{~J}$ and $40 \mathrm{~Hz}$. A continuous saline solution irrigation was applied during the enucleation and morcellation. The enucleated tissue was retrieved using a VersaCut morcellator (Lumenis Ltd.) through a 0-degree rectangular nephroscope (Karl Storz $\mathrm{GmbH} \& \mathrm{Co}$.). At the end of the operation, a 3-way 22-Fr urethral Foley catheter was inserted for continuous bladder irrigation. All retrieved tissues were weighed and examined histologically. Urethral catheters were typically removed on the first or second postoperative day after confirming clear urine color without significant gross hematuria. Patients were divided into 4 cohorts based on age as group A, 50-59 years, group B, 60-69 years, group C, 70-79 years, and group $\mathrm{D}, \geq 80$ years.

\section{Clinical Parameters}

All patients underwent a baseline evaluation including: age, body mass index, ASA score, previous BPH surgery, medications for LUTS, TRUS prostate volume, use of anticoagulation medication (aspirin, clopidogrel, warfarin, enoxaparin), and presence of urinary retention (defined as the presence of a preoperative indwelling urinary catheter or intermittent catheter- 
ization). The perioperative variables were also investigated including: enucleation time, morcellation time, enucleated specimen weight, enucleation ratio, enucleation efficiency, enucleation ratio efficiency (defined as enucleation ratio divided by enucleation time [5]), surgical pathology, changes in serum hemoglobin concentration, length of hospital stay, catheterization time, and incidence of blood transfusions. After HoLEP, the subjective and objective treatment outcomes were followed up at 2 weeks, 3 months, and 6 months postoperatively with IPSS, UFM, and PVR. Serum PSA was performed at six months. Continence was evaluated routinely at three and six months. All complications were assessed within the 1-month perioperative period and classified using the Clavien-Dindo classification [6] for surgical complications.

\section{Statistical analysis}

All parameters are represented in mean \pm standard deviation or frequency (percentage). Continuous variables were analyzed using a paired Student t-test, Mann-Whitney U-test, one-way analysis of variance (ANOVA), or Kruskal-Wallis test between the groups. Categorical variables were analyzed by the chisquare and ANOVA test. Statistical significance was considered at $\mathrm{P}<0.05$. Statistical analyses were performed by IBM SPSS Statistics ver. 20.0 (IBM Co., Armonk, NY, USA)

\section{RESULTS}

\section{Patient Demographics}

Patient demographics are shown in Table 1. Of the 579 patients, 44 (7.6\%) were in group A, 253 (43.7\%) were in group B, 244 (42.1\%) were in group C, and $38(6.6 \%)$ were in group D. The presence of hypertension was significantly higher in the older groups $(\mathrm{P}=0.007)$. The use of anticoagulation medication was significantly higher in the older groups, with the highest rate of use in group $\mathrm{D}(\mathrm{P}=0.008)$. The total prostate volume and transitional zone volume were significantly larger in the older group $(\mathrm{P}=0.024$ and $\mathrm{P}=0.002$, respectively). The baseline PSA was higher in older patients, but the difference was not significant $(\mathrm{P}=0.434)$. The ASA score was highest in group $\mathrm{D}$, with a mean of 1.8. Patients in group D had the highest incidence of urine

Table 1. Baseline characteristics

\begin{tabular}{|c|c|c|c|c|c|}
\hline Characteristic & Group A $(n=44)$ & Group B $(n=253)$ & Group C $(\mathrm{n}=244)$ & Group D $(\mathrm{n}=38)$ & P-value \\
\hline $\mathrm{BMI}\left(\mathrm{kg} / \mathrm{m}^{2}\right)$ & $24.1 \pm 3.1$ & $24.2 \pm 2.6$ & $23.7 \pm 3.1$ & $23.6 \pm 2.2$ & 0.128 \\
\hline Hypertension & $11(25.0)$ & $104(41.3)$ & $118(48.4)$ & $22(57.9)$ & 0.007 \\
\hline Diabetes mellitus & $5(11.4)$ & $46(18.2)$ & $49(20.1)$ & $8(21.1)$ & 0.562 \\
\hline Cardiovascular disease & $3(6.8)$ & $20(7.9)$ & $17(7.0)$ & $4(10.5)$ & 0.878 \\
\hline Neurologic disease & $1(2.3)$ & $30(11.9)$ & $31(12.7)$ & $6(15.8)$ & 0.201 \\
\hline Total prostate volume $(\mathrm{mL})$ & $65.6 \pm 50.1$ & $71.5 \pm 33.8$ & $68.5 \pm 38.2$ & $86.4 \pm 52.6$ & 0.024 \\
\hline Transitional zone volume (mL) & $35.1 \pm 19.6$ & $39.5 \pm 24.6$ & $40.9 \pm 28.9$ & $53.1 \pm 38.8$ & 0.002 \\
\hline Previous prostate surgery & $0(0)$ & $2(0.7)$ & $1(0.4)$ & $0(0)$ & 0.838 \\
\hline Mean ASA score & $1.5 \pm 0.5$ & $1.7 \pm 0.5$ & $1.7 \pm 0.5$ & $1.8 \pm 0.5$ & 0.006 \\
\hline Urinary retention & $3(6.8)$ & $17(6.7)$ & $22(9.0)$ & $8(21.1)$ & 0.032 \\
\hline Urinary incontinence & $3(6.8)$ & $26(10.3)$ & $42(17.2)$ & $4(10.5)$ & 0.061 \\
\hline PSA (ng/mL) & $3.5 \pm 3.2$ & $4.3 \pm 4.2$ & $3.9 \pm 4.2$ & $4.6 \pm 3.5$ & 0.434 \\
\hline \multicolumn{6}{|l|}{ Preoperative medications for $\mathrm{BPH}$} \\
\hline a blockers & $28(63.6)$ & $158(62.5)$ & $142(58.2)$ & $26(68.4)$ & 0.565 \\
\hline $5 \alpha \mathrm{RI}$ & $11(25.0)$ & $53(20.9)$ & $61(25.0)$ & $15(39.5)$ & 0.150 \\
\hline a blockers $+5 a \mathrm{RI}$ & $9(20.5)$ & $44(17.4)$ & $54(22.1)$ & $13(34.2)$ & 0.102 \\
\hline Anticoagulation & $6(13.6)$ & $56(22.1)$ & $79(32.4)$ & $13(34.2)$ & 0.008 \\
\hline
\end{tabular}

Values are presented as mean \pm standard deviation or number (\%).

Group A, age groups of 50-59 years; group B, age groups of 60-69 years; group C, age groups of 70-79 years; group D, age groups of $\geq 80$ years; BMI, body mass index; ASA, American Society of Anesthesiologists; PSA, prostate-specific antigen; $\mathrm{BPH}$, benign prostatic hyperplasia; $5 \mathrm{aRI}, 5 \mathrm{a}$ reductase inhibitor medication. 
Table 2. Perioperative outcomes

\begin{tabular}{|c|c|c|c|c|c|}
\hline Characteristic & Group A $(\mathrm{n}=44)$ & Group B $(n=253)$ & Group C $(\mathrm{n}=244)$ & Group D $(\mathrm{n}=38)$ & P-value \\
\hline \multicolumn{6}{|l|}{ Surgical outcome } \\
\hline Total operation time (min) & $52.3 \pm 30.2$ & $57.2 \pm 26.5$ & $59.7 \pm 31.0$ & $79.0 \pm 48.5$ & $0.002^{\mathrm{a})}$ \\
\hline Enucleation time (min) & $32.2 \pm 11.9$ & $37.6 \pm 17.1$ & $38.4 \pm 17.8$ & $45.3 \pm 19.2$ & $0.010^{\mathrm{a})}$ \\
\hline Morcellation time (min) & $12.0 \pm 14.8$ & $10.70 \pm 7.9$ & $11.6 \pm 10.0$ & $18.9 \pm 19.7$ & $<0.01^{\text {b) }}$ \\
\hline Enucleation weight (g) & $18.2 \pm 16.4$ & $23.5 \pm 18.9$ & $24.5 \pm 24.9$ & $34.5 \pm 28.4$ & $0.009^{\mathrm{b})}$ \\
\hline Enucleation ratio & $0.51 \pm 0.36$ & $0.53 \pm 0.25$ & $0.57 \pm 0.34$ & $0.63 \pm 0.38$ & $0.441^{b)}$ \\
\hline Enucleation efficiency (g/min) & $0.51 \pm 0.34$ & $0.61 \pm 0.64$ & $0.59 \pm 0.39$ & $0.71 \pm 0.39$ & $0.086^{\mathrm{b})}$ \\
\hline Enucleation ratio efficiency (g/mL/min) & $0.02 \pm 0.01$ & $0.02 \pm 0.02$ & $0.02 \pm 0.01$ & $0.02 \pm 0.01$ & $0.919^{b)}$ \\
\hline Catheterization time (day) & $2.1 \pm 2.7$ & $1.7 \pm 2.4$ & $2.0 \pm 2.6$ & $2.6 \pm 3.2$ & 0.268 \\
\hline Hospital stay (day) & $2.3 \pm 0.7$ & $2.3 \pm 0.7$ & $2.4 \pm 0.7$ & $2.9 \pm 1.8$ & 0.001 \\
\hline \multicolumn{6}{|l|}{ Adjuvant or salvage TUR procedure } \\
\hline Adjuvant TUC for hemostasis & $3(7.0)$ & $15(6.1)$ & $19(7.9)$ & $6(16.2)$ & 0.196 \\
\hline Salvage TURP for failed morcellation & $3(7.0)$ & $15(6.1)$ & $29(12.0)$ & $6(16.2)$ & 0.064 \\
\hline \multicolumn{6}{|l|}{ Postoperative outcome } \\
\hline Cardiovascular accident & $0(0)$ & $0(0)$ & $0(0)$ & $0(0)$ & NS \\
\hline Cerebral vascular accident & $0(0)$ & $0(0)$ & $0(0)$ & $0(0)$ & NS \\
\hline Respiratory & $0(0)$ & $0(0)$ & $0(0)$ & $0(0)$ & NS \\
\hline Fever & $0(0)$ & $0(0)$ & $0(0)$ & $0(0)$ & NS \\
\hline Acute kidney injury & $0(0)$ & $0(0)$ & $0(0)$ & $0(0)$ & NS \\
\hline PSA at 6 months $(\mathrm{ng} / \mathrm{mL})$ & $1.1 \pm 0.7$ & $1.1 \pm 0.8$ & $1.1 \pm 1.5$ & $1.9 \pm 2.5$ & 0.048 \\
\hline Surgical pathology & & & & & 0.357 \\
\hline Benign nodular hyperplasia & $40(95.2)$ & $225(96.2)$ & $204(91.5)$ & $31(88.6)$ & \\
\hline Incidental prostate cancer & $2(4.8)$ & $9(3.8)$ & $16(7.2)$ & $3(8.6)$ & \\
\hline Incidental transitional cell carcinoma & $0(0)$ & $0(0)$ & $1(0.4)$ & $0(0)$ & \\
\hline
\end{tabular}

Values are presented as mean \pm standard deviation or number (\%).

Group A, age groups of 50-59 years; group B, age groups of 60-69 years; group C, age groups of 70-79 years; group D, age groups of $\geq 80$ years; TUR, transurethral resection; TUC, transurethral coagulation; TURP, transurethral resection of the prostate; NS, not significant; PSA, prostate-specific antigen.

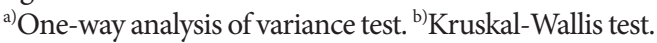

retention, occurring in 8 patients $(21.1 \%)$, and the difference was significant $(\mathrm{P}=0.032)$.

\section{Change in Perioperative Variables}

The patients' perioperative outcomes are shown in Table 2. Compared with the younger groups, the total operation time, enucleation time, and morcellation time were significantly longer and the enucleation weight was larger in the older groups ( $\mathrm{P}=0.002, \mathrm{P}=0.010$, and $\mathrm{P}<0.01$, respectively). There were no differences in the enucleation ratio, enucleation efficiency, or enucleation ratio efficiency $(\mathrm{P}=0.441,0.086$, and $\mathrm{P}=0.919$, respectively). Catheterization time was longer in the older groups, but the differences were not significant $(P=0.268)$. The length of hospital stay was the longest in group $\mathrm{D}$, with a mean of $2.9 \pm 1.8$ days $(\mathrm{P}=0.001)$. The use of the transurethral procedure for adjuvant or salvage TUR for difficult morcellation or salvage or adjuvant transurethral coagulation for bleeding control were highest in group D. The PSA level at 6 months was highest in group $\mathrm{D}$ and the difference was significant $(\mathrm{P}=0.048)$. There were no postoperative complications in all groups. The percentages of the pathological finding of incidental prostate cancer in groups A, B, C, and D were 4.8\%, 3.8\%, 7.2\%, and 8.6\%, respectively $(\mathrm{P}=0.357)$. 
Table 3. Intraoperative and postoperative complications

\begin{tabular}{|c|c|c|c|c|c|c|c|}
\hline Characteristic & Clavien-Dindo grade & Total & Group A $(n=44)$ & Group B $(n=253)$ & Group C $(n=244)$ & Group D $(n=38)$ & P-value \\
\hline \multicolumn{8}{|l|}{ Intraoperative } \\
\hline Minor capsular perforation & I & 6 & 0 & 2 & 3 & 1 & 0.655 \\
\hline Bladder mucosal injury & I & 8 & 2 & 2 & 3 & 1 & 0.216 \\
\hline \multicolumn{8}{|l|}{ Immediate postoperative } \\
\hline Recatheterization & II & 29 & 2 & 16 & 11 & 0 & 0.394 \\
\hline Blood transfusion & II & 0 & 0 & 0 & 0 & 0 & NS \\
\hline Transient SUI & I & 14 & 0 & 3 & 9 & 2 & 0.180 \\
\hline Urinary tract infection & II & 12 & 0 & 5 & 6 & 1 & 0.746 \\
\hline \multicolumn{8}{|l|}{ Late postoperative } \\
\hline Urethral stricture & IIIa & 2 & 0 & 1 & 1 & 0 & 0.954 \\
\hline Bladder neck contracture & IIIb & 5 & 0 & 3 & 2 & 0 & 0.761 \\
\hline Total sum/total patients, n (\%) & & $76 / 70$ & $4 / 4(9.1)$ & $32 / 29(11.5)$ & $35 / 32(13.1)$ & $5 / 5(13.2)$ & \\
\hline
\end{tabular}

Group A, age groups of 50-59 years; group B, age groups of 60-69 years; group C, age groups of 70-79 years; group D, age groups of $\geq 80$ years; NS, not significant; SUI, stress urinary incontinence.

Table 4. Lower tract functional outcomes

\begin{tabular}{|c|c|c|c|c|c|}
\hline Characteristic & Preoperative & 2 Weeks & 3 Months & 6 Months & P-value ${ }^{a}$ \\
\hline \multicolumn{6}{|l|}{ IPSS } \\
\hline Group A & $22.9 \pm 6.3(33)$ & $9.9 \pm 7.1(13)$ & $6.3 \pm 5.6(32)$ & $6.2 \pm 6.1(29)$ & $<0.01$ \\
\hline Group B & $19.1 \pm 7.3(222)$ & $11.4 \pm 7.8(103)$ & $7.5 \pm 5.9(204)$ & $5.3 \pm 4.8(184)$ & $<0.01$ \\
\hline Group C & $18.3 \pm 8.2(205)$ & $13.5 \pm 8.5(83)$ & $8.2 \pm 6.4(200)$ & $6.7 \pm 6.0(184)$ & $<0.01$ \\
\hline Group D & $18.5 \pm 9.2(30)$ & $14.4 \pm 10.4(9)$ & $5.9 \pm 4.2(29)$ & $5.3 \pm 6.5(29)$ & $<0.01$ \\
\hline P-value ${ }^{b)}$ & $0.014^{\mathrm{d})}$ & $0.188^{c}$ & $0.125^{\mathrm{c}}$ & $0.115^{c)}$ & \\
\hline \multicolumn{6}{|l|}{ QoL } \\
\hline Group A & $4.6 \pm 0.8(33)$ & $3.1 \pm 1.4(13)$ & $1.4 \pm 1.1(32)$ & $0.9 \pm 1.1(29)$ & $<0.01$ \\
\hline Group B & $4.2 \pm 1.3(222)$ & $2.5 \pm 1.6(103)$ & $1.6 \pm 1.4(204)$ & $1.3 \pm 1.4(184)$ & $<0.01$ \\
\hline Group C & $4.1 \pm 1.4(205)$ & $2.7 \pm 1.8(83)$ & $1.7 \pm 1.5(200)$ & $1.4 \pm 1.3(184)$ & $<0.01$ \\
\hline Group D & $4.1 \pm 1.6(31)$ & $2.6 \pm 2.1(9)$ & $1.3 \pm 1.3(29)$ & $1.1 \pm 1.1(29)$ & $<0.01$ \\
\hline P-value ${ }^{b)}$ & $0.429^{\mathrm{d})}$ & $0.697^{\mathrm{d})}$ & $0.336^{c)}$ & $0.196^{c)}$ & \\
\hline \multicolumn{6}{|l|}{$\mathrm{Qmax}(\mathrm{mL} / \mathrm{sec})$} \\
\hline Group A & $8.6 \pm 4.4(43)$ & $22.4 \pm 11.0(42)$ & $25.1 \pm 10.9(30)$ & $23.6 \pm 13.4(25)$ & $<0.01$ \\
\hline Group B & $9.0 \pm 4.3(239)$ & $21.9 \pm 10.3(243)$ & $24.3 \pm 11.4(191)$ & $21.4 \pm 11.4(154)$ & $<0.01$ \\
\hline Group C & $8.9 \pm 4.8(225)$ & $17.9 \pm 9.3(217)$ & $21.0 \pm 15.1(191)$ & $19.7 \pm 9.8(166)$ & $<0.01$ \\
\hline Group D & $9.3 \pm 4.5(33)$ & $16.0 \pm 8.7(36)$ & $16.8 \pm 8.2(30)$ & $17.1 \pm 7.4(27)$ & $<0.01$ \\
\hline P-value ${ }^{\mathrm{b})}$ & $0.942^{c)}$ & $<0.01^{c)}$ & $0.004^{c)}$ & $0.077^{\mathrm{c})}$ & \\
\hline \multicolumn{6}{|l|}{ PVR (mL) } \\
\hline Group A & $68.5 \pm 128.0(43)$ & $22.8 \pm 41.4(42)$ & $17.9 \pm 36.0(32)$ & $22.8 \pm 26.6(27)$ & 0.044 \\
\hline Group B & $66.4 \pm 91.3(243)$ & $20.7 \pm 36.5(243)$ & $14.3 \pm 34.3(196)$ & $13.6 \pm 19.9(155)$ & $<0.01$ \\
\hline Group C & $71.9 \pm 105.5(236)$ & $24.1 \pm 35.1(217)$ & $22.7 \pm 41.2(196)$ & $16.1 \pm 24.3(166)$ & $<0.01$ \\
\hline Group D & $71.6 \pm 72.3(34)$ & $40.8 \pm 49.3(37)$ & $36.6 \pm 57.2(30)$ & $38.7 \pm 49.7(28)$ & 0.01 \\
\hline P-value ${ }^{\text {b) }}$ & $0.943^{c)}$ & $0.129^{\mathrm{d})}$ & $0.150^{\mathrm{d})}$ & $0.078^{\mathrm{d})}$ & \\
\hline
\end{tabular}

Values are presented as mean \pm standard deviation (number).

Group A, age groups of 50-59 years; group B, age groups of 60-69 years; group C, age groups of 70-79 years; group D, age groups of $\geq 80$ years; IPSS, International Prostate Symptom Score; QoL, quality of Life; Qmax, maximal flow rate; PVR, postvoid residual urine.

${ }^{a)} \mathrm{P}$-value represents the comparison between preoperative results and postoperative 6-month results. ${ }^{\text {b) }} \mathrm{P}$-value represents the difference among the 4 groups. ${ }^{c}$ One-way analysis of variance test. ${ }^{\mathrm{d})}$ Kruskal-Wallis test. 

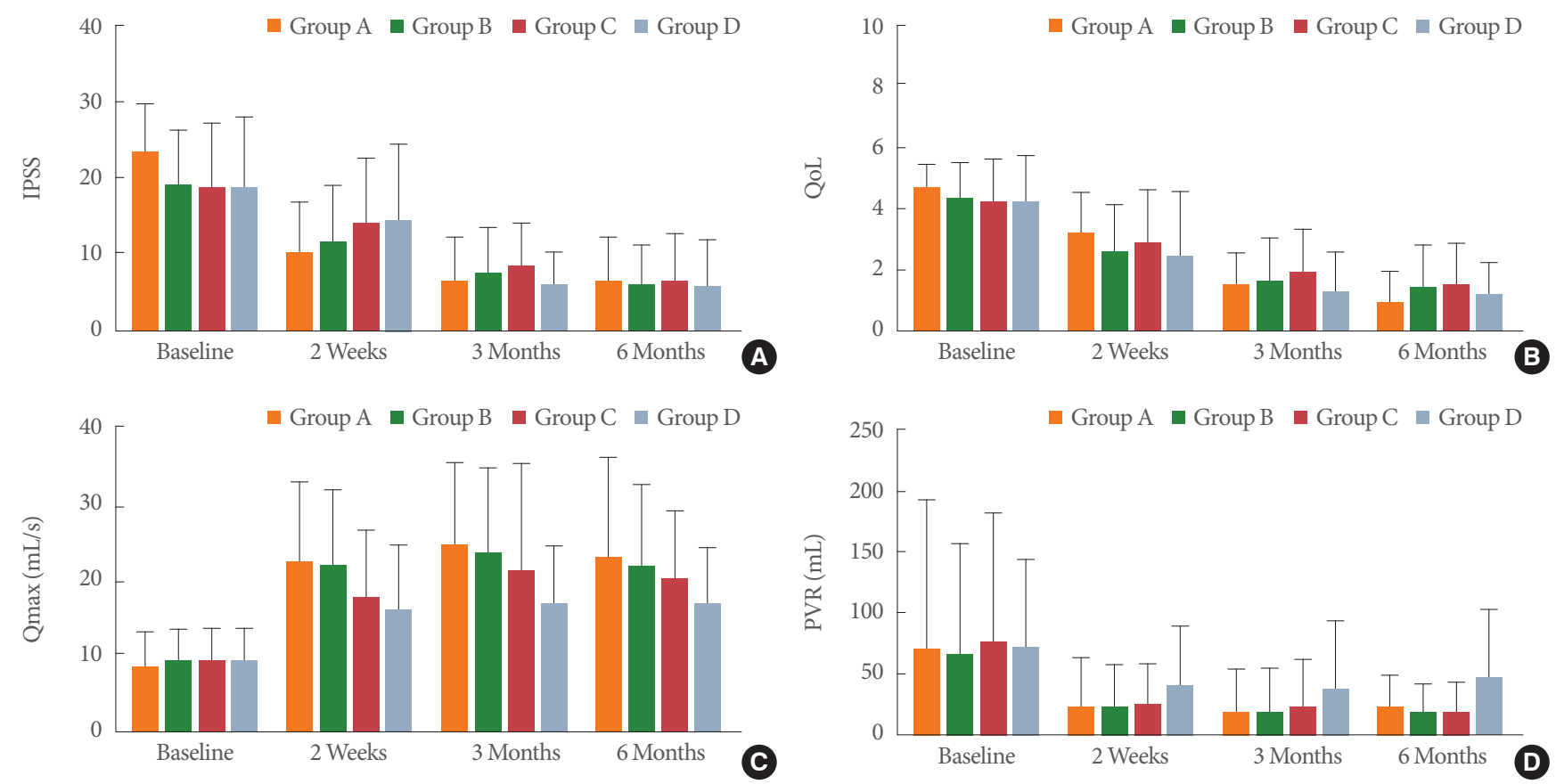

Fig. 1. Age-specific changes of subjects International Prostate Symptom Score (IPSS; A) and quality of life (QoL; B), and in objective rate of urinary flow (Qmax; C) and postvoid residual (PVR; D) volume from baseline to various follow-up assessments. Group A, age groups of 50-59 years; group B, age groups of 60-69 years; group C, age groups of 70-79 years; group D, age groups of $\geq 80$ years.

\section{Change in Clinical Outcomes}

The intraoperative and postoperative complications were similar in all groups (Table 3). The incidence of significant complications (Clavien-Dindo grade $\geq$ III) was rare in all groups. There were no grade IV or V complications.

All groups showed improvement in their LUTS at the postoperative 6-month follow-up, compared to the preoperative results (Table 4, Fig. 1A-D). Only IPSS had a significant difference among the groups in the preoperative evaluation. Patients in group D had a significant lower Qmax than the other groups at 2-week $(\mathrm{P}<0.01)$ and 3-month follow-up $(\mathrm{P}=0.004)$. There were no significant differences in PVR or QoL at all time points $(\mathrm{P}>0.05)$.

\section{DISCUSSION}

LUTS is a very common urogenital disorder in older men. The aging population of Korea has led to an increasing number of surgical operations in elderly patients. The increasing evidence indicates that HoLEP has become a new gold standard method for the treatment of LUTS [3]. The advantages of HoLEP over OP and TURP, such as the lower amount of blood loss, particularly in patients receiving anticoagulation therapy, lack of trans- urethral resection syndrome, use of normal saline as the irrigant, and shorter convalescence are well documented. However, surgery in old patients is a special challenge.

Surgical morbidity showed a linear increase across all age groups. Patients with BPH, who are older than 70 years and have significant comorbidities, are considered as high-risk patients [7]. Uchida et al. [8] found age was an independent predictor of both morbidity and the need for a blood transfusion after TURP in a large cohort study of 3,861 patients. Matani et al. [9] reported that early and late complications occurred in $25.9 \%$ and $13.2 \%$ of patients who underwent TURP, respectively. However, a cohort study of 311 patients who underwent HoLEP procedures reported that the overall morbidity, hospital stay, and 1-year functional outcomes were similar among the $50-59,60-69,70-79$, and $\geq 80$-year groups [10]. In our study, hospital stay was significant longer in patients $\geq 80$ years. The longer incidences of hospital stay in our series are attributable to the longer catheterization time in group $\mathrm{D}$ compared with other group patients. Furthermore, it can be explained by the difference in the selection criteria of candidates for surgery and the relatively small number of patients in group $\mathrm{D}$. Patients in group D had a longest catheterization time of $2.6 \pm 3.2$ days, although there was no significant difference among these groups. 
This is probably a consequence of higher preoperative urinary retention in group D (21.1\%).

As Marmiroli et al. [11] reported, the prevalence of hypertension, a history of surgery, and other complications such as cardiovascular disease and neurologic disease increased in older patients significantly. Several baseline characteristics of the patients were significantly different among the 4 groups, including the presence of hypertension and urinary retention, total prostate volume, transitional zone volume, mean ASA score, and the number of patients using anticoagulation medications, which increased with age. The larger enucleation weight in group D correlated with their longer total operation time, enucleation time, and morcellation time. These results were consistent with those previously reported [12]. Oral anticoagulant use independently affects TURP outcome, with longer hospital stay, and higher rates of bleeding, blood transfusion, late hematuria, and thromboembolic events [11]. As Mmeje et al. [10] reported, the overall blood transfusion rate was highest in patients $\geq 80$ years ( $11 \%$ ) who underwent HoLEP. In this study, no patient needed a blood transfusion during surgery. This difference may be due to the different in preoperative hemoglobin levels and other multifactorial reasons. The baseline serum PSA levels increased with advancing age $(3.5 \pm 3.2,4.3 \pm 4.2,3.9 \pm 4.2$, and $4.6 \pm 3.5 \mathrm{ng} / \mathrm{mL}$ in groups $\mathrm{A}, \mathrm{B}, \mathrm{C}$, and $\mathrm{D}$, respectively), although the difference was not significant. Furthermore, we found that patients in group D had a higher incidence of incidental prostate cancer, compared with the other groups; however, the difference was not significant.

In this study, the Clavien-Dindo classification [6] of surgical complications was used. Overall, there were 76 surgery-related complications in 579 patients (13.1\%). However, all of these complications were mild and transient. The incidence of complications in all groups was not significantly different. ClavienDindo grade III complications occurred in 7 patients (1.2\%), and the incidence was highest in group B. Higher grade events were not observed. Our results appeared different from that of previous studies owing to the difference in the follow-up period. In our study, the maximum follow-up period was 6 months. There were 6 occurrences (1.0\%) of minor capsular peroration and 8 cases (1.4\%) of bladder mucosal injury during surgery.

All patients in this study showed an improvement in the functional outcomes after HoLEP (Fig. 1A-D). There was a gradual improvement in IPSS, QoL, Qmax, and PVR over the 6-month follow-up period. The lowest improvement in Qmax and PVR was observed in the patients of group D over the 6-month follow-up period, although there was no significant difference among the four groups. It is assumed that detrusor contractility decreased in patients aged $\geq 80$ years due to a prolonged obstruction, resulting in a longer recovery time. It is also important that all postoperative data included all patients regardless of their preoperative voiding ability. In this study, there was no mortality during the perioperative or follow-up period.

The results of the present study have shown that HoLEP is safe and effective, regardless of patient age. Nadu et al. [13] reported that patients $>80$ years of age who underwent either TUPR or OP had a significantly higher overall postoperative complication rate, compared with those aged $<65$ years $(39 \%$ vs. $22 \%, \mathrm{P}<0.05)$. However, in this study, similar complications were observed in the various groups, despite patients aged $\geq 80$ years had a higher presence of hypertension, TRUS prostate volumes, ASA scores, urinary retention, and anticoagulation use during the perioperative period. Functional outcomes at 6 months were also similar to the younger groups, even though more than 1 of 5 of the patients aged $\geq 80$ years had urinary retention before surgery.

There were several limitations in this study. First, a possible selection bias may have existed in this study. It should be noted that the patients who were considered to be adequate candidates for surgery after clinical evaluation were included in the study. Thus, the complication rates were likely reduced because HoLEP was not performed in elder patients with more adverse clinical conditions. Second, in this study, the surgical outcomes were assessed with short-term follow-up of 6 months. The longterm follow-up results would be more informative to investigate outcomes in patients who underwent HoLEP. However, in our opinion, the surgical outcomes could be reasonably assessed at 6 months. Finally, in this series, all patients were operated by a single surgeon. The data from multicenters are and further are needed to generalize the findings of this study. Despite these limitations, to the best of our knowledge, this is the first study to date on the morbidity and outcomes after HoLEP with an adequate number of subjects in Asians according to age groups.

In conclusion, the findings of this study indicated that HoLEP for BPH can be performed in patients older than 80 years with an acceptable perioperative morbidity and good functional results. HoLEP is a safe and effective treatment for elderly patients with $\mathrm{BPH}$. 


\section{REFERENCES}

1. El-Husseiny T, Buchholz N. Transurethral ethanol ablation of the prostate for symptomatic benign prostatic hyperplasia: long-term follow-up. J Endourol 2011;25:477-80.

2. Lepor H, Kazzazi A, Djavan B. a-Blockers for benign prostatic hyperplasia: the new era. Curr Opin Urol 2012;22:7-15.

3. Holman CD, Wisniewski ZS, Semmens JB, Rouse IL, Bass AJ. Mortality and prostate cancer risk in 19,598 men after surgery for benign prostatic hyperplasia. BJU Int 1999;84:37-42.

4. Michalak J, Tzou D, Funk J. HoLEP: the gold standard for the surgical management of BPH in the 21(st) Century. Am J Clin Exp Urol 2015;3:36-42.

5. Jeong CW, Oh JK, Cho MC, Bae JB, Oh SJ. Enucleation ratio efficacy might be a better predictor to assess learning curve of holmium laser enucleation of the prostate. Int Braz J Urol 2012;38:362-71.

6. Dindo D, Demartines N, Clavien PA. Classification of surgical complications: a new proposal with evaluation in a cohort of 6336 patients and results of a survey. Ann Surg 2004;240:205-13.

7. Lusuardi L, Hruby S, Janetschek G. New emerging technologies in benign prostatic hyperplasia. Curr Opin Urol 2013;23:25-9.
8. Uchida T, Ohori M, Soh S, Sato T, Iwamura M, Ao T, et al. Factors influencing morbidity in patients undergoing transurethral resection of the prostate. Urology 1999;53:98-105.

9. Matani Y, Mottrie AM, Stockle M, Voges GE, Fichtner J, Hohenfellner R. Transurethral prostatectomy: a long-term follow-up study of 166 patients over 80 years of age. Eur Urol 1996;30:414-7.

10. Mmeje CO, Nunez-Nateras R, Warner JN, Humphreys MR. Agestratified outcomes of holmium laser enucleation of the prostate. BJU Int 2013;112:982-9.

11. Marmiroli R, Antunes AA, Reis ST, Nakano E, Srougi M. Standard surgical treatment for benign prostatic hyperplasia is safe for patients over 75 years: analysis of 100 cases from a high-volume urologic center. Clinics (Sao Paulo) 2012;67:1415-8.

12. Piao S, Choo MS, Wang Y, Lee YJ, Bae J, Oh SJ. Clinical and pathological characteristics of hard nodules resistant to morcellation during holmium laser enucleation of the prostate. Int Neurourol J 2015;19:90-8.

13. Nadu A, Mabjeesh NJ, Ben-Chaim J, Kaver I, Matzkin H, Greenstein A. Are indications for prostatectomy in octogenarians the same as for younger men? Int Urol Nephrol 2004;36:47-50. 